\title{
TACTIC: A New Detector for Tracking in Low Energy Nuclear Astrophysics
}

\author{
K.A. Chipps ${ }^{* a, 1}$, P.A. Amaudruz ${ }^{2}$, L. Buchmann ${ }^{2}$, P. Bruskiewich ${ }^{2}$, S.P. Fox ${ }^{1}$, \\ B.R. Fulton ${ }^{1}$, U. Hager ${ }^{2}$, A.M. Laird ${ }^{1}$, P. Machule ${ }^{2}$, L. Martin ${ }^{2}$, R. Openshaw ${ }^{2}$, \\ G. Ruprecht ${ }^{2}$, A.C. Shotter ${ }^{2}, 3$, P. Walden ${ }^{2}$, M. Walter ${ }^{2}$ \\ ${ }^{a}$ E-mail: kc612@york.ac.uk \\ ${ }^{1}$ Department of Physics, University of York, Heslington, York, UK \\ ${ }^{2}$ TRIUMF Laboratory, Vancouver, BC, Canada \\ ${ }^{3}$ School of Physics and Astronomy, University of Edinburgh, Edinburgh, UK
}

In nuclear astrophysics, detection of low energy reaction products can often prove difficult with conventional techniques such as silicon detectors. Time Projection Chambers (TPCs) and active target detectors have helped to alleviate these problems, but are often rate limited, making measurements of low reaction cross sections nearly impossible. To this end, the TRIUMF Annular Chamber for Tracking and Identification of Charged particles (TACTIC) detector has been designed and built by an international collaboration between TRIUMF and the University of York. Specific technical details are discussed, and preliminary results are shown.

11th Symposium on Nuclei in the Cosmos

19-23 July 2010

Heidelberg, Germany.

\footnotetext{
*Speaker.
} 


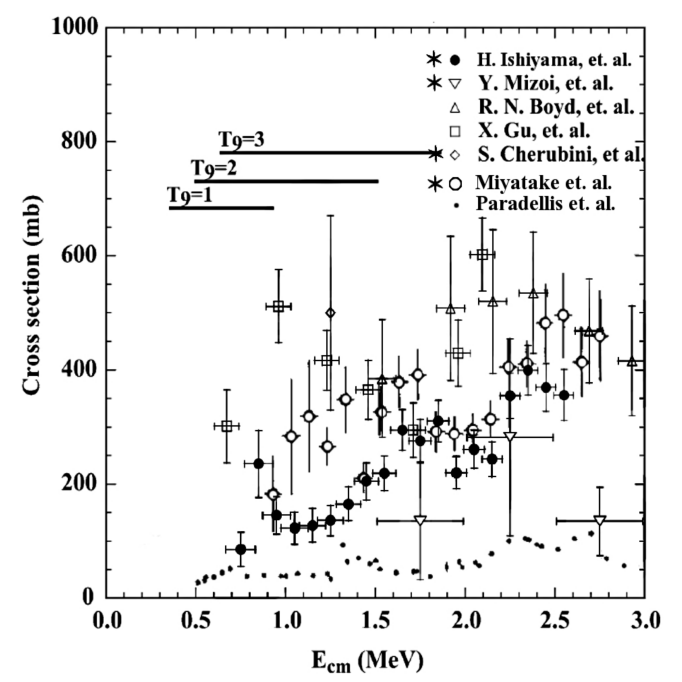

Figure 1: ${ }^{8} \mathrm{Li}(\alpha, \mathrm{n}){ }^{11} \mathrm{~B}$ cross section data, adapted from Ref. [3].

\section{Astrophysical Motivation}

The ${ }^{8} \mathrm{Li}(\alpha, \mathrm{n}){ }^{11} \mathrm{~B}$ reaction could be an important step in non-standard Big Bang nucleosynthesis [1]. The reaction, while having surprisingly little effect on ${ }^{11} \mathrm{~B}$ abundance, can have a tremendous effect on heavier elements $\left({ }^{12} \mathrm{C}\right.$ and onward), as most of these are produced when the ${ }^{8} \mathrm{Li}$ abundance is large and thus reactions on it are of significant importance. Additionally, the ${ }^{8} \operatorname{Li}(\alpha, n){ }^{11} \mathrm{~B}$ reaction may also be important as an initial step in the r-process, getting past the "mass-8 gap" and acting as a secondary reaction sequence to the triple-alpha process [2]. A plot of the current cross section data as taken from Ref. [3], as well as relevant Gamow windows for different temperature regimes, is shown in Figure 1.

Previous measurements of the ${ }^{8} \operatorname{Li}(\alpha, n){ }^{11} \mathrm{~B}$ reaction have met with difficulty. A time-reversed reaction measurement was limited only to the ground state of ${ }^{11} \mathrm{~B}$ [4], but theory [5] indicated excited states in ${ }^{11} \mathrm{~B}$ were likely to play a significant role in the reaction rate. Direct measurements (see, for example, Refs. [1,3]) have been limited by beam intensity and detection methods. A more thorough discussion may be found in Ref. [6].

\section{Detector Specifications}

TACTIC, the TRIUMF Annular Chamber for Tracking and Identification of Charged particles, is a cylindrical, segmented-anode, active-target time-projection chamber (TPC) [7, 8, 9, 10]. The geometry mirrors the kinematics of the reactions studied, and utilizes a "blind" center region which traps the ionization created by the beam particles and allows much higher beam intensities than typical TPCs. High-gain, low cost-per-channel preamplifiers and digital electronics are used to instrument the 480 anode pads, and a new graphical analysis tool for the data is being developed and implemented. Amplification of the small signals is accomplished using a Gas Electron Multiplier 


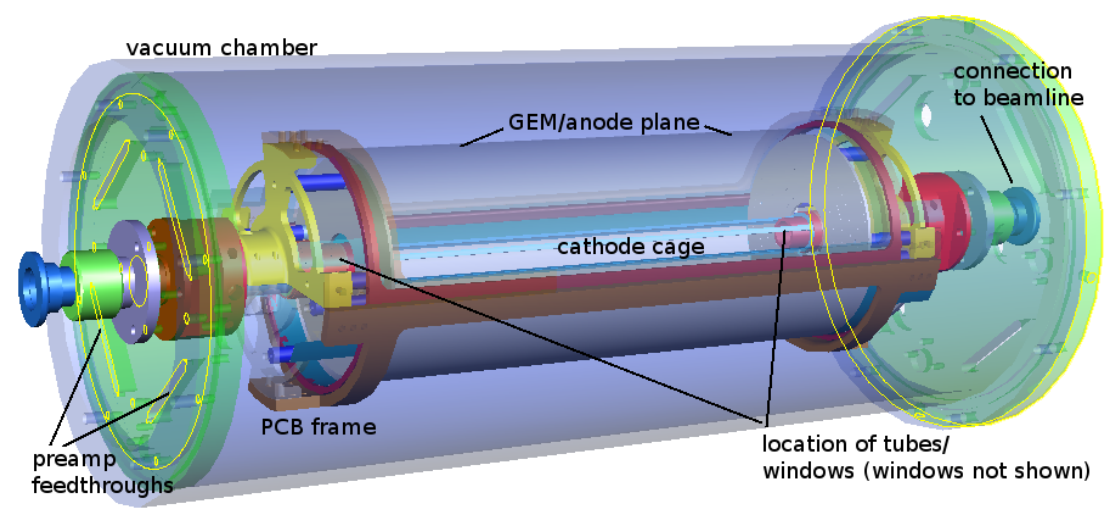

Figure 2: 3D CAD drawing of the complete TACTIC detector. Dimensions are given in the text; the active volume is the region inside the PCB anode frame. The gap between the GEM and anode plane is not visible due to scale.

(GEM) in place of a traditional Frisch grid. The fill gas, a $\mathrm{He}-\mathrm{CO}_{2}$ mixture, provides both particle detection and a homogeneous, variable-thickness target for studying reactions on alphas; thin mylar windows mounted on tubes at either end of the detector contain the gas mixture and can be used to vary the active target length from 47 to $250 \mathrm{~mm}$ (differing lengths of tube can be used). A three-dimensional CAD drawing of the detector is shown in Figure 2. A prototype was built by a consortium of researchers from Daresbury Laboratory and the University of York in the UK and TRIUMF laboratory in Canada.

Inside of the active volume of the TACTIC prototype are three regions, as demonstrated in Figure 3. Reactions take place in Region 1 (the target region), inside the cathode cage. The cathode cage is comprised of two concentric cylinders (10.575 and $12 \mathrm{~mm}$ radius) of tensioned 20 micron gold-coated tungsten wire, held at different voltages such that ionization from the beam is confined to within Region 1. This allows for higher beam currents than traditional TPCs, as the detector is not saturated with signals from the beam itself. Initial tests indicated that pile-up only occurred after about $5 \times 10^{7}$ pps of beam [7], a significant improvement over the roughly $10^{4}$ pps limit of standard TPCs and ion chambers. Signals arising from "good" events within this region, however, are lost, so the reaction vertex of a track must be reconstructed. The TACTIC cathode is generally held at a large negative bias, such that the anode pads are held at ground.

Region 2 serves as the drift region, where particles of interest (reaction products) ionize the active-target fill gas and the subsequent drift electrons move toward the Gas Electron Multiplier (GEM) foil (50 mm radius). GEMs have been used mainly in high-energy physics applications (see, for example, Refs. [11, 13]), but because of their high gain are ideally suited for use in TACTIC, where low reaction energies lead to small signals from ionization of the fill gas. The design of the GEM is such that the electric field is "funneled" into the transfer/collection region (Region 3). Electrons from the drift region effectively create an avalanche inside of this funneled GEM field, increasing the gain (electrons produced per unit track length) by factors of up to $\sim 10^{4}$, depending on the ionizing particle, fill gas, pressure and GEM bias. These electrons are collected onto 480 anode pads (only a few millimeters outside the GEM foil), the TACTIC anode being 

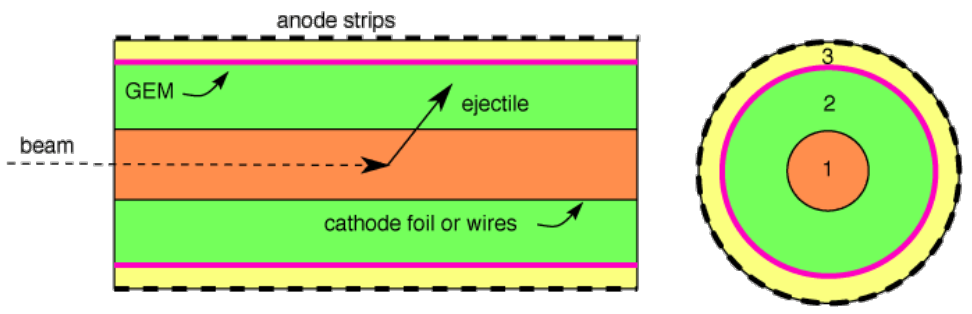

Figure 3: The three regions inside the active volume of TACTIC (not to scale). Region 1 (the target region), inside of the cathode cage, is the active target; the cathode cage serves to confine the ionization from the beam within this region. Region 2 is the drift region, separated from Region 3 (the transfer/collection region) by a Gas Electron Multiplier (GEM) foil.

segmented both in $\mathrm{z}$ and phi, and read out into specially-designed preamplifiers (more technical specifications may be found in Ref. [7]).

The TACTIC detector relies upon low cost-per-channel, high-gain preamplifier chips to magnify the signals from the 480 anode pads. Sixteen preamplifier cards, with 32 channels per card, are used to read out the signals from the anode pads as well as signals from the different GEM segments. These preamplified signals are piped into several VF48 digital data acquisition modules [14], which digitize the waveforms. The VF48s can be programmed to either write out full waveforms, or to provide pulse height and timing information as extracted via various programmable algorithms.

A new analysis software tool is being written and tested by TACTIC collaborators which will allow for 3D graphical reconstruction of particle tracks. For instances when full waveforms were recorded by the VF48 modules, these waveforms can be visualized and analyzed. Raw amplitude (pulse height) and time signals are converted into energy and radius, taking into account the TACTIC operational parameters, allowing for track reconstruction. Particle identification can then be achieved via techniques utilizing kinematics and energy loss, either summed or by sector.

\section{Preliminary Results}

Previous attempts to measure the ${ }^{8} \mathrm{Li}(\alpha, \mathrm{n})^{11} \mathrm{~B}$ reaction cross section have met with difficulty, so a direct study of the reaction using TACTIC was proposed and carried out at TRIUMF in Vancouver in June of 2009. This was the first time TACTIC had ever been used with radioactive beam. Preliminary analysis of the data shows scattered $\mathrm{CO}_{2}$ from the fill gas, scattered ${ }^{8} \mathrm{Li}$ from the beam, and a large peak resulting from one of the two alphas from the decay of the ${ }^{8} \mathrm{Li}$ beam (via breakup of ${ }^{8} \mathrm{Be}$ ), as well as possible ${ }^{11} \mathrm{~B}$ events; see Figure 4. Analysis of this recent data is ongoing to determine absolute cross sections, which can then be compared with earlier results.

\section{Conclusion}

The TACTIC detector has been used with its first radioactive beam, and the preliminary results are promising: specifically, the hardware works, the particle tracking is successful, and the new analysis software is nearly complete, but improvements will continue to lower the thresholds and 


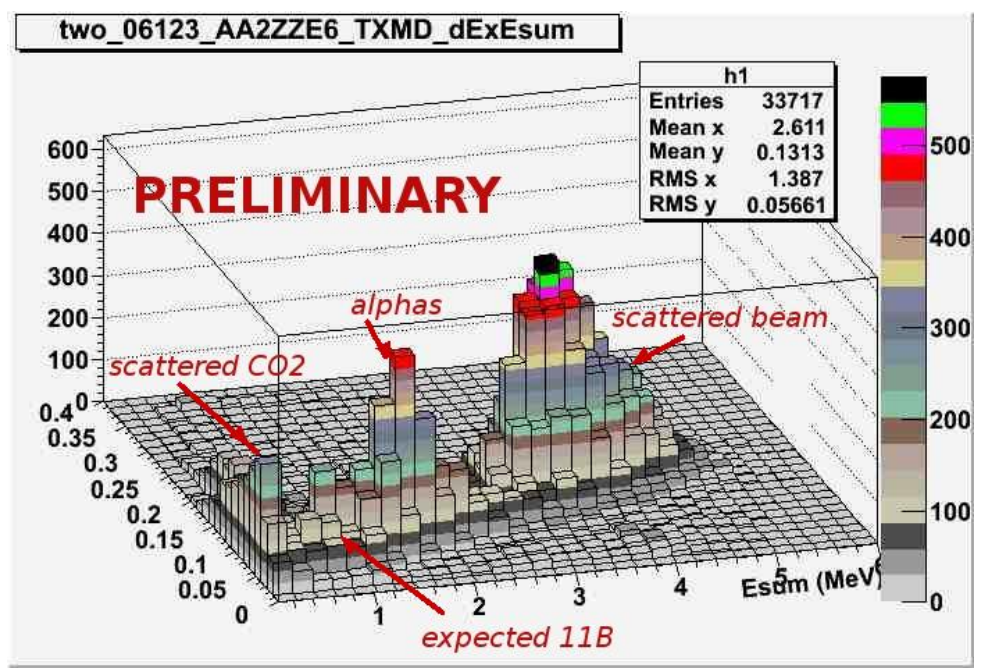

Figure 4: $\mathrm{A} \Delta \mathrm{E}$ vs $\mathrm{E}$ plot for preliminary $12 \mathrm{MeV}{ }^{8} \mathrm{Li}(\alpha, \mathrm{n}){ }^{11} \mathrm{~B}$ data. Locations of the scattered beam and target gas, the alpha particles from decay of the beam, and the expected location of ${ }^{11} \mathrm{~B}$ recoils are indicated.

improve resolution. Modifications to the existing preamplifiers and data acquisition system will improve detector performance, and ongoing data analysis will elucidate further successes and areas for optimization. In the future, TACTIC will be used to study more reactions, including ${ }^{7} \mathrm{Be}+\mathrm{p}$ elastic scattering and ${ }^{7} \mathrm{Li}\left({ }^{3} \mathrm{He},{ }^{4} \mathrm{He}\right){ }^{6} \mathrm{Li}[15]$.

\section{References}

[1] R. Boyd et al., Comments, Nucl. Part. Phys. 22, 47 (1996)

[2] M. Terasawa et al., Astrophys. J. 562, 470 (2001)

[3] H. Ishiyama et al., Phys. Lett. B 640, 82 (2006)

[4] T. Paradellis et. al, Z. Phys. A 337, 211 (1990)

[5] T. Rauscher et al., Phys. Rev. C45, 1996 (1992)

[6] P.D. Mumby-Croft, PhD Thesis, University of York (2009)

[7] S.P. Fox et al., Proceedings of the Int. Nuc. Phys. Conf., Vancouver, BC, Canada, submitted (2010)

[8] L. Martin et al., J. Phys.: Conf. Series 202, 012040 (2010)

[9] A.M. Laird et al., Nucl. Instrum. Methods Phys. Res. A573, 306 (2007)

[10] G. Ruprecht et al., Eur. Phys. J. A 27, s01, 315-320 (2006)

[11] A. Balla et al., Nucl. Instrum. Methods Phys. Res. A604, 23 (2009)

[12] H. Fenker et al., Nucl. Instrum. Methods Phys. Res. A592, 273 (2008)

[13] A. Ishikawa et al., arXiv:0710.0205v1 [physics.ins-det ] (2007)

[14] TRIUMF DAQ website, http://daq-plone.triumf.ca/HR/VME/VF 48/VF 48, accessed Aug 2010

[15] TACTIC website, tactic.triumf.ca, accessed Aug 2010 